REVISTA PROYECCIONES № 15: 75-82

Diciembre 1988 - ISSN 0716-0917

\title{
ON SOME NONLINEAR EQUATIONS
}

IOANNIS K. ARGYROS*

Abstract.

A new method for finding large solutions of quadratic equations is presented.

Key words and phrases. Quadratic equation Banach space, large solutions.

A.M.S. subject classification codes. $46(B 15), 65$.

Introduction.

Consider the quadratic equation

$$
x=y+\lambda B(x, x)
$$

* Department of Mathematics, New Mexico State University. Las Cruces, New Mexico 88003, U.S.A. 
in a Banach space $X$, where $y \varepsilon X$ is fixed, $\lambda$ is a positive number and $B$ is a bounded symmetric bilinecr oferator on $X[1],[5],[7]$.

The above equation has already been studied in [1], [2], [3], [5] and the references there. Briefly, it is known that if

$$
4 \lambda|| B|| \cdot|| y||<1
$$

then a small solution $x$ of (1) exists (i.e., a solution tending to 0 with y) such that

$$
|| x|| \leq \frac{1 \sqrt{1-4 \lambda|| B|| \cdot|| y||}}{2 \lambda|| B||}
$$

The problem of finding a not necessarily small solution $x$ of (1) is of great importance. The best known approach to this problem is the application of the Newton's Kantorovich iteration [6]

$$
x_{n+1}=x_{n}-\left(I-2 \lambda B\left(x_{n}\right)\right)^{-1}\left(y+\lambda B\left(x_{n}, x_{n}\right)-x_{n}\right), \quad n=0,1,2, \ldots
$$

for some $x_{0} \in X$. The application of the above iteration, however, does not necessarily guarantee that the obtained solution $x$ is not the small solution.

Here motivated by the solution of the real quadratic equation and the work in [5] and [6], we seek a solution $x$ of (1) expressed as

$$
x=\frac{1}{\lambda} v+\sum_{n=0}^{\infty} \lambda^{n} x_{n} \text {, }
$$


where, $v, x_{n} \varepsilon X, n=0,1,2, \ldots$ are to be specified. Under certain assumptions on $v$ we show that if (2) holds the solution $x$ of (1) given by (5) is such that

$$
|| x|| \geq \frac{1+\sqrt{1-4 \lambda|| B|| \cdot|| y||}}{2 \lambda|| B||}
$$

We now state the main results.

Theorem. Assume:

(a) there exists $\vee \varepsilon X$ satisfying

$$
B(v, v)=v, v \neq 0
$$

and such that the linear operator $(I-2 B(v))^{-1}$ exists or $X$.

(b) Let $k$ denote the norm of $(I-2 B(v))^{-1}$ and set

$$
\begin{aligned}
& x_{0}=(I-2 B(v))^{-1}(y) \\
& x_{n}=\sum_{j=0}^{n-1}(I-2 B(v))^{-1} B\left(x_{j}, x_{n-j-1}\right), \quad n=1,2, \ldots
\end{aligned}
$$

with

$$
4 \lambda k^{2}|| B|| \cdot|| y||<1 \text { and }|| B|| \neq 0 \text {. }
$$


Then there exists a solution $x$ of (1) given by (5) and satisfying

$$
|| x|| \leq \frac{1}{\lambda}|| v||+\frac{1-\sqrt{1-4 \lambda k^{2}|| B|| \cdot|| y||}}{2 \lambda|| B|| \cdot k}
$$

Proof. As in [5], formal substitution of (5) into (1) and equa tion of like powers of $\lambda$ shows that if $x$ is a solution then $v, x_{n}$ ' $n=0,1,2, \ldots$ must be giben by (7) and (8).

The real series

$$
\frac{1}{\lambda}|| v||+\sum_{n=0}^{\infty} \lambda^{n} z_{n},
$$

where

$$
\begin{aligned}
& z_{0}=k|| y|| \\
& z_{n}=\sum_{j=0}^{n-1} k|| B|| z_{j} z_{n-j-1} .
\end{aligned}
$$

obviously dominates the series given by (5). Moreover, by (9), we have

$$
\sum_{n=0}^{\infty} \lambda^{n} z_{n}=\frac{1-\sqrt{1-4 \lambda k^{2}|| B|| \cdot|| y||}}{2 \lambda|| B|| \cdot k} .
$$

Therefore, the series given by (5) converges to a solution $x$ of (1) satisfying (10) and the proof is completed. 
We now prove the existence of a not small solution. For simplicity we take $\lambda=1$.

Proposition. If the hypotheses of the theorem are satisfied, $k$ is such that

$$
0<k \leq 1 \text { and } 1-4|| B|| \cdot|| y||>0 \text {. }
$$

then there exists a solution $x$ of (1) given by (5) and satisfying

$$
\|x\| \geq \frac{1+\sqrt{1-4|| B|| \cdot|| y||}}{2|| B||} .
$$

Proof. The solution $x$ of (1) given by (5) is guaranteed by the theorem. Hence, it is enough to show (11). By (5) we have

$$
\begin{aligned}
|| x|| & \geq\|v\|-\left\|\sum_{n=0}^{\infty} x_{n}\right\| \\
& \geq\|v\|-\sum_{n=0}^{\infty}|| x_{n}|| \\
& \geq\|v\|-\frac{1-\sqrt{1-4 k^{2}|| B|| \cdot|| y||}}{2|| B|| k}
\end{aligned}
$$

If $v$ is a nonzero solution of (7), then

$$
\|v\|=|| B(v, v)|| \leq\|B|| \cdot\| v \|^{2} \text {. }
$$


Therefore,

$$
|| v|| \geq \frac{1}{\| B||}
$$

Now, (12), because of (13), becomes

$$
\|x\| \geq \frac{1}{\|B\|}-\frac{1-\sqrt{1-4 k^{2}|| B|| \cdot|| y||}}{2|| B|| k}
$$

By (14), to show (11), it is enough to show

$$
\frac{(2 k-1)+\sqrt{1-4 k^{2}|| B|| \cdot|| y||}}{2|| B|| \cdot k} \geq \frac{1+\sqrt{1-4|| B|| \cdot|| y||}}{2|| B||}
$$

After the simplifications showing (15) becomes easily equivalent to showing

$$
0<k \leq 1
$$

which is true by hypothesis and the proof is completed.

Note that in the case of the real quadratic equation

$$
r=\alpha+\lambda \beta r^{2}
$$

where $\alpha=|| y||$ and $\beta=|| B||$ equality is achieved (3) and (6). The solutions are then given by 


$$
\begin{aligned}
r^{-} & =\frac{1-\sqrt{1-4 \lambda \alpha \beta}}{2 \lambda \beta} \\
& =\sum_{n=0}^{\infty} \lambda^{n} z_{n} \\
& =\sum_{n=0}^{\infty} 2^{n} \lambda^{n} \alpha^{n+1} B^{n} \frac{13 \ldots(2 n-1)}{12 \ldots(n+1)} .
\end{aligned}
$$

and

$$
r^{+}=\frac{1}{\lambda \beta}-r^{-}
$$

Finally, note that for $v=0$ in theorem 2, we obtain the result in [5]. 


\section{REFERENCES.}

1 Argyros, I.K. Quadratic equations and applicctions to Chandrasekhar's and related equations. Bull. Austral. Math. Soc. Vol. 32, № 2, (1985), pp. 275-292.

2 Kantorovich, L.V. Functional analysis and applied mathematics. Uspe ki Mat. Nauk, (1948), pp. 89-185.

3 Kelley,C. T. Approximation of solutions of some quadratic integral equations in transport theory. Journal of Integrcl Equations, 4, (1982), pp. 221-237.

4 McForland, J. An iterative solution of the qucdratic equation. Proc. Amer. Math. Soc., 9, (1958), pp. 824-830.

5 Rall, L.B. Quadratic equations in Banach space. Rerd. Circ. Math. Palermo, 10, (1961), pp. 314-332.

6 ----. Solution of abstract polynomial equations by iterative methods. University of Wisconsin, Technical report № 892, (1968).

7 -.-. Nonlinear functional analysis and applications. Academic Press, New York, (1971). 